\title{
Thermal Study of Anhydrous and Hydrated Forms of Olanzapine
}

\author{
Cristina Cavallari ${ }^{1}$, Adamo Fini ${ }^{1 *}$ and Beatriz Pérez-Artacho Santos
}

${ }^{1}$ Department FABIT, University of Bologna, Bologna, Italy

${ }^{2}$ Departamento de Farmacia y Tecnologia Farmaceutica, Universidad de Granada, Granada, Spain

\begin{abstract}
Anhydrous and hydrated forms of olanzapine were obtained by crystallization using different experimental pathways and analysed by differential scanning calorimetry and thermogravimetric techniques. The unsolvated form I could be prepared by direct crystallization from a limited number of solvents; the form II could obtained only after desolvation of selected solvates. At thermal analysis the form I and II of olanzapine demonstrated to be polymorphs; while the form III appears to be rather a solvate of undefined stoichiometry. The hydrated forms were obtained both from fully and partially aqueous media, and only with difficulty by direct crystallization from water, due to very poorly solubility in water of the drug. Depending on the experimental conditions, hydrates of different composition could be obtained that, on de-hydration, leave the de-hydrated forms of olanzapine, possibly via-(partial) amorphization.
\end{abstract}

Keywords: Olanzapine; Anhydrous forms; H/solvate forms; Thermal analysis

\section{Introduction}

The formation of solvates or, hydrates if the solvating species is water, by inclusions of crystallization solvent molecules into the crystal lattice, as well the possible occurrence of different polymorphic forms, makes intriguing and often difficult the pre-formulation study for the correct description of the solid state of a drug. All these aspects can have effects on the physical properties of the drug, such as solubility, hygroscopicity, drying properties and chemical stability and crystallinity. This is the case of solid olanzapine, an important modern antipsychotic agent, whose manufacturing process is complicated by its ability to form polymorphs, hydrates and other solvates: a number of unsolvated crystalline forms, polymorphic forms, numerous hydrates, alcoholates, mixed solvates have been obtained, patented and described, especially by X-ray crystallography [1].

The olanzapine molecule consists of a 1,5-diazepine ring fused with a benzene, at one side, and with a methylthiophene ring at the other one; an additional non-planar N-methylpiperazine fragment is also present. The molecular structure is thus configured in a butterflylike shape, being about $120^{\circ}$ the dihedral angles between the side fused rings and the central one. The crystal lattice olanzapine is described consisting in dimers arranged in columns that contain channels that can be filled with guest molecules: the inclusions of small organic molecules into a crystal lattice of solvates provides stabilization of the crystal by their hydrogen bond donor and acceptor ability that generates intermolecular links between neighbouring columns [2,3].

These facts can explain the facility to form solvate for olanzapine, when crystallizes from solvents and the necessity to test the nature of the solid state of the final crystals thus obtained, as a function of the solvent and their technological properties, since the presence of a second species in the crystal lattice affects, beside the chemical and physical properties, also other properties, such as processability and formulations as well as bioavailability and therapeutical efficiency of the drug.

Moreover solvate forms, inadvertently generated during crystallization, may also be the key to obtain the different polymorph forms observed for olanzapine: therefore attention must be paid on the polymorphism and hydrate/solvate formation, when this drug is processed to optimize the physical presentation of the final solid [4].
As a consequence, before considering pharmaceutical formulations that contain solid crystalline olanzapine, well documented characteristics of the physicochemical properties of this drug are required and determination of optimal and controlled conditions to obtain solid of known properties represent a significant challenge for the pharmaceutical technologist.

The determination of a solvated compound carried out by the resolution of the crystallographic structure starting from a monocrystal often is difficult to get. We explored another way based on thermal and thermogravimetric analysis to evaluate stability and thermal behaviour of a series anhydrous and hydrate forms. Unexpected results allowed the description of a general behaviour for olanzapine in the solid state when obtained after dehydration or desolvation.

\section{Materials and Methods}

Olanzapine was a gift: the sample was crystallized for purification by cooling an anhydrous ethyl acetate solution that gives the stable form I used for crystallization experiments throughout this paper.

\section{Preparation of olanzapine forms I, II and III}

Form I: Olanzapine was dissolved in anhydrous boiling ethyl acetate: the solution on cooling to ambient temperature let crystallize the form I, that was isolated by filtration, dried in vacuo at $50^{\circ} \mathrm{C}$ and identified by the DSC thermogram (melting endotherm at $197^{\circ} \mathrm{C}$ ). The same was obtained by crystallization also from acetone, ethyl ether and toluene.

Form II: a) Olanzapine was dissolved in boiling aqueous ethanol $96 \%(\mathrm{v} / \mathrm{v})$ : the solution on cooling to ambient temperature let crystallize a solvate that was isolated by filtration, air-dried at room temperature.

*Corresponding author: Adamo Fini, Department FABIT, Via San Donato 15 , 40127 Bologna, Italy, Tel: +39 051 2095655; Fax: +39 051 2095652; E-mail: adamo.fini@unibo.it

Received May 15, 2013; Accepted May 23, 2013; Published May 27, 2013

Citation: Cavallari C, Fini A, Pérez-Artacho Santos B (2013) Thermal Study of Anhydrous and Hydrated Forms of Olanzapine. Pharm Anal Acta 4: 237. doi:10.4172/2153-2435.1000237

Copyright: (c) 2013 Cavallari C, et al. This is an open-access article distributed under the terms of the Creative Commons Attribution License, which permits unrestricted use, distribution, and reproduction in any medium, provided the original author and source are credited. 
The ethanol solvate thus formed was desolvated at $50^{\circ} \mathrm{C}$ in the oven and identified by DSC thermogram by the typical peak profile associated to a polymorph transition before the melting endotherm at $197^{\circ} \mathrm{C}$.

b) Olanzapine crystallizes from dichloromethane as a solvate (loss of weight $3.58 \%$, corresponding to a stoichiometry $2: 1$ ); after mild heating the form II was identified by the melting endotherm (Tpeak $\left.183^{\circ} \mathrm{C}, \Delta \mathrm{H}=25.10 \mathrm{Jg}^{-1}\right)$.

c) Olanzapine was suspended in dichloromethane and stirred for a week at room temperature; the slurry was filtered, dried at air and, analysed by DSC, demonstrated to be a partial solvate (loss of weight: $1.59 \%)$. The presence of the form II was evident by a large endotherm (Tpeak $181^{\circ} \mathrm{C} ; \Delta \mathrm{H}=45.60 \mathrm{Jg}^{-1}$ ).

Form III: This form was obtained according to [4]. Olanzapine dissolves in chloroform on heating; the solution was cooled to ambient temperature and added of $n$-hexane to start crystallization. The solid precipitate was isolated by vacuum filtration, washed with $n$-hexane and dried. We tested also alternative methods using different solvents for olanzapine (acetone, toluene) but the same anti-solvent (n-hexane).

\section{Preparation of olanzapine hydrate forms}

Method 1: Olanzapine form I was suspended in water at ambient temperature and added of a dilute solution of hydrochloric acid; the solution containing protonated olanzapine was then added of a dilute solution containing sodium carbonate. The solid precipitate was isolated by vacuum filtration, washed with water to neutrality and airdried.

Method 2: Olanzapine form I was suspended in water at ambient temperature and stirred for a week. The solid product was isolated by vacuum filtration, washed with water, and air-dried. This method allows the formation of the Di-hydrate D [4].

Alternatively the system was heated up to boiling point, filtered the undissolved material, and the left evaporate the solvent until crystallization started: the precipitate was filtered and air dried.

Method 3: Olanzapine form I was dissolved in ethyl acetate and toluene $(1: 10 \mathrm{v} / \mathrm{v})$ at about $60^{\circ} \mathrm{C}$. The solution was then added of water and cooled to room temperature. The solid precipitate was isolated by vacuum filtration, washed with water and air-dried.

The preparation was also repeated using a different ethyl acetate/ toluene $(1: 17 \mathrm{v} / \mathrm{v})$ ratio. These methods were proposed to prepare $\mathrm{Di}$ hydrate $B$ and $E[4]$.

Method 4: Olanzapine form I was crystallized from ethyl acetate, previously saturated with water; or from water saturated with ethyl acetate (solubility of ethyl acetate in water $8.7 \% \mathrm{v} / \mathrm{v}$; solubility of water in ethyl acetate $3.3 \% \mathrm{v} / \mathrm{v}$ at $20^{\circ} \mathrm{C}$ ). The same was repeated with toluene saturated by water. The solid precipitate was filtered at reduced pressure, washed with the solvent and dried. More suitable to this purpose could appear acetone: its full miscibility with water at all the proportions allows crystallization of the hydrate form of olanzapine in more controlled conditions.

Higher Hydrate: This sample was prepared according to [4]. Olanzapine was dissolved in boiling dichloromethane. Water was added when the solution was at room temperature. The precipitate was filtered and dried.

The samples obtained by the different methods were examined by DSC and TG techniques.

\section{Thermal methods of analysis}

Differential Scanning Calorimetry (DSC): DSC traces were recorded with an automatic thermal analyser system (Mettler $\left.821^{\mathrm{e}}\right)$. The data processing system (Mettler $821^{\mathrm{e}} / 500 / 847$ thermo-cryostat) was connected to the thermal analyser. Sealed and holed aluminium pans were used for the experience for all the samples. Indium (melting point $156.6^{\circ} \mathrm{C}$ ) was used to calibrate the instruments. The thermograms were run at a heating rate of $10^{\circ} \mathrm{C} / \mathrm{min}$, from 30 to $320^{\circ} \mathrm{C}$ under a nitrogen purge of $20 \mathrm{~mL}$ per minute. A mass, not exceeding $5 \mathrm{mg}$, was measured into aluminium pans with a small pinhole in the lid. The peaks were identified by the peak temperature.

Thermogravimetric analysis (TGA): Loss of solvent from the crystals was characterized by thermogravimetric analysis (TGA) with a Mettler Toledo automatic thermal analyser system TGA/SDTA851 ${ }^{\mathrm{e}} /$ $\mathrm{SF} / 1100)$. TGA traces were recorded at heating rates of $10^{\circ} \mathrm{C}$ per minute under a nitrogen purge. Samples with masses between 1 and $10 \mathrm{mg}$ were analyzed using open alumina crucibles; mass loss (\%) was calculated from TG curves, based on the mass of the original sample. The weight percentage changes allowed the determination of the stoichiometry of the different hydrates/solvates, assuming that the solvate was formed only by the crystallization solvent.

\section{Results and Discussion}

It has been reported that olanzapine exists in two anhydrous forms which are clearly distinguishable by X-ray powder diffractometry: one of them, defined as anhydrous olanzapine form II, was not well suited for commercial use in pharmaceutical formulations [5]; while the anhydrous form I of the drug, is stable and adapted for commercial use in pharmaceutical formulations and can be prepared both utilizing solvates of olanzapine formed when it crystallizes from the lower alcohol, such as methanol; or by direct crystallization by selected solvents.

Many pioneering works (especially patents) on the olanzapine solid state, mainly containing only qualitative results, are indicative of an intense research work regarding the different solid state phases of olanzapine. Recently it was summarizes that the drug exists in three different anhydrides, three dehydrated forms, a higher hydrate and an undisclosed number of solvates and mixed solvates [4]. The structure of some of these forms have been described by a number of papers using sophisticated analysis such as FT-Raman, infrared and near infrared $[3,6], \mathrm{X}$ ray diffraction $[1,7] ;{ }^{13} \mathrm{C}$ and ${ }^{15} \mathrm{~N} \mathrm{CP} /$ MAS NMR [4] and ${ }^{1} \mathrm{H}-{ }^{13} \mathrm{C}$ NMR spectroscopy [8]. In front of the simplicity of thermal analysis to study the solid state of the drugs, this technique, as investigation tool of the olanzapine solid state, was employed only in few papers $[9,10]$.

Using DSC and TGA in this paper we re-examined some of the unsolvated or hydrated forms that can be prepared for olanzapine to get easy and rapid information about the solid form obtained following different experimental pathways and the forms that remain after desolvation of different solvates of olanzapine.

\section{Anhydrous forms}

Form I: The unsolvated olanzapine form I can be obtained by direct crystallization from a few solvents such as ethyl acetate, ethyl ether, acetone and toluene and possibly other solvents non considered here. The thermogram profiles of the form I crystallized from anhydrous ethyl acetate contains only an endothermic peak centred at $197^{\circ} \mathrm{C}$, related to the melting of the sample (melting $\Delta \mathrm{H}=143 \mathrm{Jg}^{-1}$ ), not associated to weight loss, suggesting a notable stability of the molecule 
even at high temperature: the baseline of the thermogram is perfectly horizontal and does not show any thermal anomaly up to the melting.

Unsolvated olanzapine form I can be also obtained after desolvation of, e.g., methanol or other solvates, which leave this form after mild heating.

Form II: The form II could not be obtained by direct crystallization, but only after desolvation of solvates obtained by a range of solvents: the thermogram shown in Figure 1 refers to the form obtained from the ethanol solvate after desolvation. The thermogram profile of this solvate shows the presence of a desolvation endotherm (Tpeak at $114^{\circ} \mathrm{C} ; \Delta \mathrm{H}=174 \pm 10 \mathrm{Jg}^{-1}$ ), then a typical profile of a polymorph transition (melting endotherm of the metastable form rapidly followed by a crystallization exotherm of the stable form) and the melting endotherm of the stable form (Tpeak at $197^{\circ} \mathrm{C}$ ). After treatment in the oven at $50^{\circ} \mathrm{C}$ for a few days, desolvation of the ethanol solvate (loss of weight $=11.27 \%$, corresponding to a monosolvate) was complete and the thermogram (Figure 1A) maintains the characteristic shape of a polymorph transition with an endothermic peak (at about $184^{\circ} \mathrm{C}$ ) and an exothermal signal (at $185^{\circ} \mathrm{C}$ ) partially fused to the final melting endotherm found at $197^{\circ} \mathrm{C}$, perfectly overlapping that of the form I.

The occurrence of the form II in an olanzapine sample is thus related to the presence of this typical profile in the thermogram and the $\Delta \mathrm{H}$ value of the first melting endotherm could offer useful information. The thermal profiles can be kept as references for the identification of the two forms I and II and particularly of the form that remains after desolvation of the different solvates.

This point concerning the form II, however, requires some discussion.

- In some case the polymorph transition appears only as a weak trace in the thermogram of the olanzapine solvates (Figure 1B); in others the first melting endotherm is larger and associated to higher $\Delta \mathrm{H}$ values than that observed in the case of the ethanol solvate. This fact allows the suspicion that the form II could not exist before, rather it appears after desolvation. The process in fact is accompanied by amorphization of the mass, since the desolvation endotherm is always followed by an exotherm. Therefore it could be hypothesized that during the reorganization of the crystal lattice, the form II appears in competition with the form I. This idea agrees with what previously reported [9] that X-ray powder diffraction diagrams, taken as a function of temperature, showed coexistence of both form I and form II in the temperature range $160-180^{\circ} \mathrm{C}$ of the ethanol solvate, that is after desolvation.

- The ratio between the two forms in the final mass depends on the experimental conditions and/or the nature of the escaping solvent during desolvation: the amount of the form II could be estimated by its melting $\Delta \mathrm{H}$. The thermogram of olanzapine solvate crystallized from dichloromethane shows after desolvation (Figure 1C) a melting endotherm of the form II of similar values of surface areas that are unaffected by the mild heating for desolvation: the last melting endotherm $\Delta \mathrm{H}=195$ $\mathrm{Jg}^{-1}$ ) suggests a complete conversion to the final form that is represented only by the form I.

- The form II is quite stable: it was reported [9] that the form II maintains stable at room temperature for long periods of time (ca. 1 year), since the spontaneous inter conversion process in the solid state occurs with transformation kinetics quite slow: the transition to the stable form occurs in fact only at quite high temperature and with "surprising immediacy" probably catalysed by the presence of "undetectable amounts of form I in the bulk of form II, and which would act as seeds triggering the re-crystallization" process [9]

- Also heating the samples at relatively low temperatures to desolvation process does not affect this transition: however the desolvation process is probably responsible of the appearance of the form II but is unable to produce a pure form II, that will be always accompanied by the form I. It could be concluded that a pure form II of olanzapine cannot be isolated and separately studied.

- The notation form I and form II as used in [4] contradicts that of the original patent [5], which labelled them exactly the opposite. It takes into account the fact that I is the stable form with the highest melting point. According to this the form II should represent the metastable one: however, due to the high thermal stability of olanzapine and that no signs of reversibility of the polymorph transition could be observed (the two forms represent a dimorphic monotropic system [9]), the term metastable for olanzapine form II should be considered with attention, since the drug is thermally stable even at temperatures relatively far from the ambient one, even though it was reported that the form II results unsuitable for commercial use, as it discolours in the presence of air [10]. Some paper published on this topic maintains the term form I for the metastable one $[1,2]$ and this could create confusion.

Form III: The form III, as proposed in [4], was obtained by addition of $\mathrm{n}$-hexane to a solution of olanzapine in chloroform and was recognized by ${ }^{13} \mathrm{C} \mathrm{CP} / \mathrm{MAS}$ NMR spectrum in the solid state [4].

The precipitate obtained following these suggestions was dried at air and analysed by DSC and TGA. The thermogram demonstrated that the sample is a solvate (Figure 2A) (weight loss 3.94\%, in the temperature range $60-100^{\circ} \mathrm{C}$ ), whose composition does not correspond to a simple stoichiometric formula. Examination of the solvate by EDS techniques indicates the presence of $\mathrm{Cl}$ atom inside the sample that can be due only to chloroform molecules: it can be assumed that the process produces a (partial) chloroform solvate, such as the direct crystallization from pure chloroform (weight loss $14.1 \%$ for a complete 1:2 solvate)

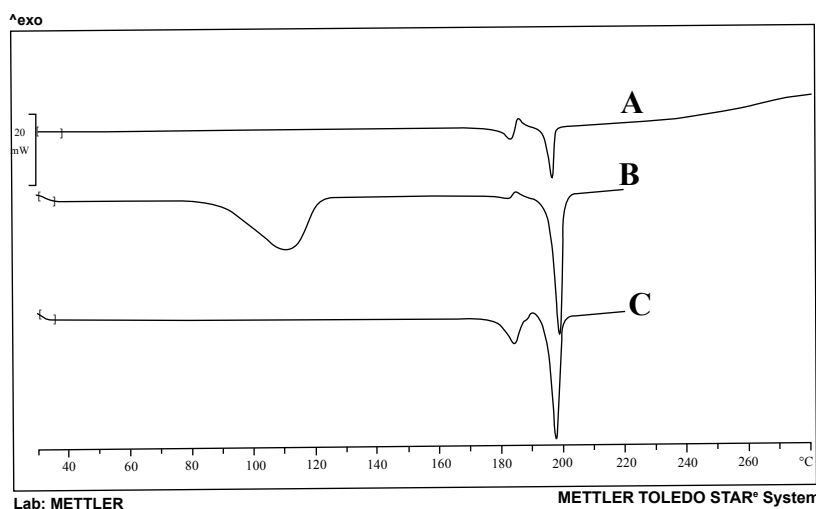

Figure 1: Olanzapine form II. Thermogram profiles of: A) ethanol solvate after 15 day heating in the oven at $50^{\circ} \mathrm{C} ; \mathrm{B}$ ) the solvate obtained after suspension in tetrahydrofuran of olanzapine form I for 10 days at room temperature; C) dichloromethane solvate after one day heating in the oven at $80^{\circ} \mathrm{C}$. 
After storage in a dessiccator the desolvation endotherm progressively disappears, leaving only a thermogram profile typical of the Form I.

DSC thus demonstrated not to enable the identification of a form III different from the other two forms of olanzapina I and II: it allowed only to put in evidence some differences of this form with respect to the forms I and II here listed.

- The composition of the solvated olanzapine form III appears to depend on the experimental conditions in terms of weight loss on desolvation, as well as the position and shape of the desolvation endotherm in the DSC thermogram, that in some case is represented by a weak trace (Figures 2A, 2B and 2C).

- Thermal anomaly in the thermogram (Figure $2 \mathrm{~A}$, at about $180^{\circ} \mathrm{C}$ ) suggests the contemporary presence of a foreign form, possibly the form II, according to what reported [4] that mixtures of forms I, II, and/or III are routinely recognized by foreign peaks in X-ray diffractograms or resonance splits in the SSNMR spectra.

- Differently to other forms that demonstrated to be quite stable even after melting up to $220^{\circ} \mathrm{C}$, the thermogravimetric profile of the form III (Figure 2D) indicates weight loss, starting from $160^{\circ} \mathrm{C}$ and appears to continue even above $200^{\circ} \mathrm{C}$, that is, after the olanzapine form I melting: in the same temperature range the thermogram did not display any thermal events that could be associated to the weight loss.

It is not clear the occurrence of the form III following the solvent/ antisolvent method. We tested also other solvent/antisolvent pair. A sample obtained using dichloromethane displays a thermogram similar to previous ones, with the presence of a weak desolvation endotherm; while using toluene, as a solvent unable to produce solvate, the thermogram display only the formation of the form I, after the addition of the n-hexane (thermograms not shown)

- DSC is a rather invasive technique and, during the temperature scanning, thermal events occur that can destroy or level the structural differences, especially when they are small and may not be critical. From this point of view, DSC analysis demonstrated useless to put in evidence subtle structural differences among the different olanzapine forms, recognized

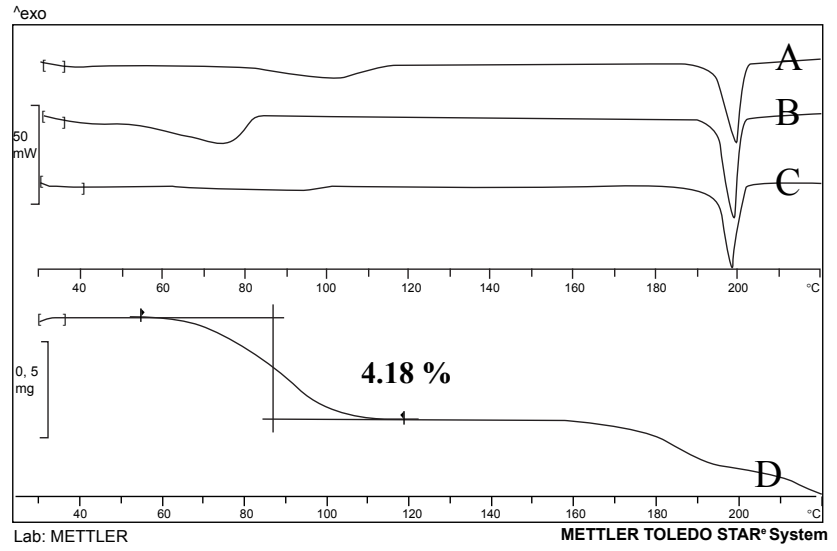

Figure 2: Olanzapine form III. Thermogram profiles of: A), B), C) samples of the form III obtained in the same experimental conditions. D) Thermogravimetric profile of the sample A). by different analytical techniques. In the present case no information could be found concerning the melting with conversion to the more stable polymorph occurring in the solid state reported for the form III by [4].

\section{Hydrated forms}

Due to the low solubility of water of olanzapine it has not been possible to directly crystallize the hydrate form from water. Olanzapine in fact is a hydrophobic molecule $(\log \mathrm{P}=2.199$ at $\mathrm{pH}=9)$ and therefore poorly soluble in its de-protonated form $(0.0165 \mathrm{~g} / \mathrm{L}$ at $\mathrm{pH}=9)$ Solubility increases at $\mathrm{pH}$ lower than $\mathrm{pKa}$ (7.37-4.69): $87.4 \mathrm{~g} / \mathrm{L}$ and correspondingly $\log \mathrm{P}$ decreases $(0.3)$ at $\mathrm{pH}=5$ [12].

Therefore to obtain the hydrate form we also tested different methods, as those reported in the literature.

\section{Hydrates from water}

Here methods were employed only using aqueous environment.

Crystallization from water: Olanzapine was suspended in a large volume of boiling water; after filtration the excess water was evaporated under reduced pressure until precipitate appears that was filtered and examined: this proved to be the only method enabling to directly obtain the hydrate form from an aqueous solution without any transformation.

Suspension in water: Olanzapine powder was suspended in water under continuous stirring at room temperature. After a week the slurry was filtered and dried demonstrating at thermal analysis that hydration occurred. Probably the dynamic equilibrium established in the saturated solution allows continuous dissolution/crystallization of the suspended material leading to the formation of the hydrate form; the same was observed in the case of many organic solvents with the formation of respective solvates. This sample was considered the form $\mathrm{D}$ of the ref. [4].

Acid/base reaction in water: In this method olanzapine, which is weak base, was first brought into aqueous solution by the addition of a dilute aqueous solution of $\mathrm{HCl}$ and then treated dropwise with a dilute solution of sodium carbonate, resulting in The precipitation of the hydrate.

In the presence of $75 \% R U$ - Olanzapine demonstrated not to hydrate when a powdered sample was kept for a month in a closed container at $75 \% \mathrm{RU}$, generated by the presence of a $\mathrm{NaCl}$ saturated aqueous solution: the thermogram did not show any signal of formation of the hydrate.

Figure 3 shows the DSC and TG profiles for the hydrate form obtained with the acid/base reaction. The thermogram (Figure 3A) shows two endotherms centered, the first at $100^{\circ} \mathrm{C}$ and the second at $197^{\circ} \mathrm{C}$ that signals the melting of the anhydrate form and this last part of the thermogram overlaps with that of a pure sample of reference form I. The first endotherm has a rounded broad and asymmetric profile that covers a large temperature range of about 50 degrees (54 to $\left.107^{\circ} \mathrm{C}\right)$ and corresponds to a dehydration process $\left(\left(\Delta \mathrm{H}=293 \mathrm{Jg}^{-1}\right)\right.$. Soon after the dehydration endotherm, a small exotherm peak $\left(124^{\circ} \mathrm{C}\right)$ can be observed, as if dehydration left the sample amorphous and recrystallization occurs on heating, driving the appearance of foreign forms, in relative amounts depending on the experimental condition: see for instance the endotherm trace at $184^{\circ} \mathrm{C}$ (form II?) evident in the sample dehydrated in the oven at $100^{\circ} \mathrm{C}$ for two days (Figure $3 \mathrm{~B}$ ). This peak is better appreciable when the hydrate is obtained by the mixed solvent method. 


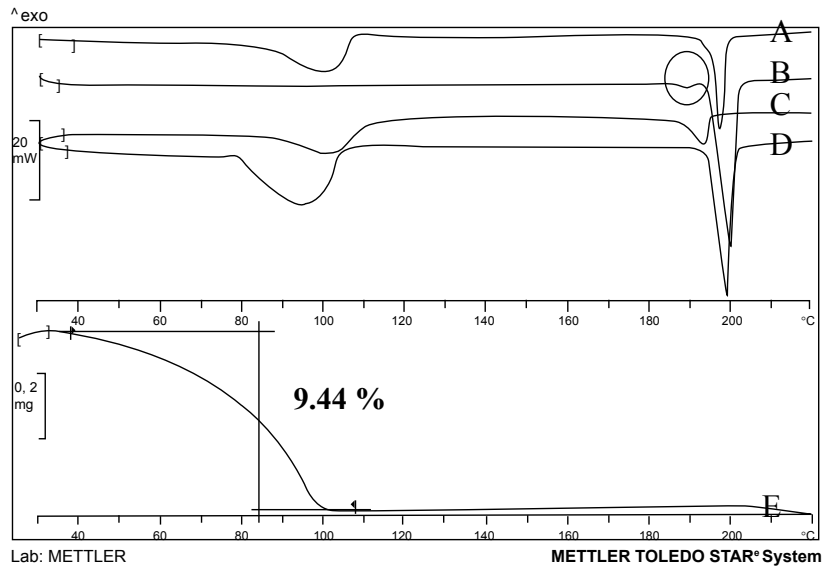

Figure 3: Olanzapine hydrate form. Thermogram profile of the hydrate: $A$ obtained from an acid/base reaction; B) after dehydration in the oven at $80^{\circ} \mathrm{C}$ for a week; C) directly obtained from water; D) obtained by suspension in water for a week at room temperature. D) Thermogravimetric profile of the sample obtained by the acid/base reaction.

The temperature peak of the dehydration endotherm shifts from 100 to $108^{\circ} \mathrm{C}$ on heating in the oven, decreases its area surface that is practically cancelled after 5 day-heating in the oven at $40^{\circ} \mathrm{C}$ : the final form is that of olanzapine form I: after this event the sample shows a remarkable stability, because the baseline of the thermogram does not show other discontinuity until about the final melting endotherm.

The thermogravimetric profile appears rather asymmetric and shows a weight loss of $9.82 \%$ (Figure $3 \mathrm{E}$ ), which begins almost as soon as heating starts at about $40^{\circ} \mathrm{C}$ and ends sharply at $116^{\circ} \mathrm{C}$, reaching $35 \%$ just before the onset of the de-hydration endotherm. A stoichiometric calculation allows the estimate that the weight loss corresponds to the presence of two molecules of water per molecule of olanzapine. Loss of weight was observed also after storage of the hydrate over silica gel in dessiccator: this can explain that the water content, as determined by TGA, is a little lower than the theoretical one (10.34\%), since the sample was left to dry at air and room temperature for one day. The continuity of the dehydration curve in the TGA profile and the tendency to dehydration reveal that the water molecules in the hydrate olanzapine are not tied too strongly to the crystal lattice, indicate low stability of the hydrate form and suggests to store in humidity-controlled container for a safe conservation of the hydrate form. However a sample of the form I, kept in a box at 75\% RU, showed no tendency to hydrate, even after one month.

Samples directly crystallized from water or obtained by suspension in water display thermograms little different: in the first case the dehydration endotherm is centered at lower temperature and the samples resulted practically amorphous. The second case that represents the Di-hydrate D of the [4] demonstrates that hydratation of olanzapine occurs by prolonged contact with water, despite its low solubility (Figure 3C and 3D).

\section{Hydrates from aqueous organic solvents}

A second process involves the use of mixed solvents.

Crystallization from aqueous organic solvents: This method proved to be suitable to obtain crystallized olanzapine hydrate: the solvents tested to this purposes do not interact with olanzapine that crystallizes unsolvated from them. The samples obtained from solvents immiscible with water offer thermograms overlapping, showing the exotherm (Figure 4A and 4B); in the case of the sample obtained from acetone the dehydration endotherm is found at lower temperature and no exotherm is evident but a weak trace of a thermal event can be found about $185^{\circ} \mathrm{C}$ (Figure 4C). The dehydration endotherm followed by an exotherm supports the idea that the first process is accompanied by a disorganization of the crystal lattice that, however, recovers its organization soon after that dehydration is terminated [11].

Crystallization from aqueous solvents, which demonstrate to be practically immiscible with water, appears suitable with small samples, since the amount of the available water is often uncertain and could be very limited, even though in these conditions at a high thermodynamic potential. The use of acetone appears better for larger samples since, being this solvent totally miscible with water, crystallization could be carried out in the presence of well fixed and varying amount of water.

Crystallization from mixed solvent: The Figure 5 shows the thermograms of olanzapine samples crystallized from some methanol/ water mixtures at various concentrations $\mathrm{v} / \mathrm{v}$; for comparison also the thermograms of samples obtained from pure water (Figure 5A) and pure methanol (Figure 5E) are shown: olanzapine forms with these solvent a Di-hydrate and a monosolvate [12]. The comparison of these profiles reveals some specific differences. In all the instances it could be appreciated the presence of a desolvation endotherm, whose shape changes passing from water to mixtures rich in methanol: up to $60 \%$ water in the mixture the endotherm resembles that of a simply hydrate sample. Starting from the concentration $40 \% \mathrm{v} / \mathrm{v}$ water the endotherm shows the typical profile of the methanol solvate: particularly useful as a marker is the endotherm portion centred at about $140^{\circ} \mathrm{C}$, whose area surface increases a the methanol concentration increases (Figures $5 \mathrm{~B}-5 \mathrm{E}$ ) and is absent in the profiles $5 \mathrm{~A}$ and $5 \mathrm{~B}$, ranging from 10 to $36 \mathrm{Jg}^{-1}$ for the for the pure methanol. These data suggest the formation of a mixed methanol/water solvates, when the system is formed by comparable volumes of the two solvents. In some cases it is appreciable the trace of a weak endotherm associated to the form II, while after the desolvation always emerges form I. It was not possible to state the formation of mixed solvates water/methanol from these thermograms, although it is reported their formation by crystallization [13]

Precipitation with non-solvents: Literature offers two examples of precipitation with a non solvent: olanzapine was first dissolved in an organic medium and then precipitated by addition of water; the methods reported used solvents immiscible with water: ethyl acetate/

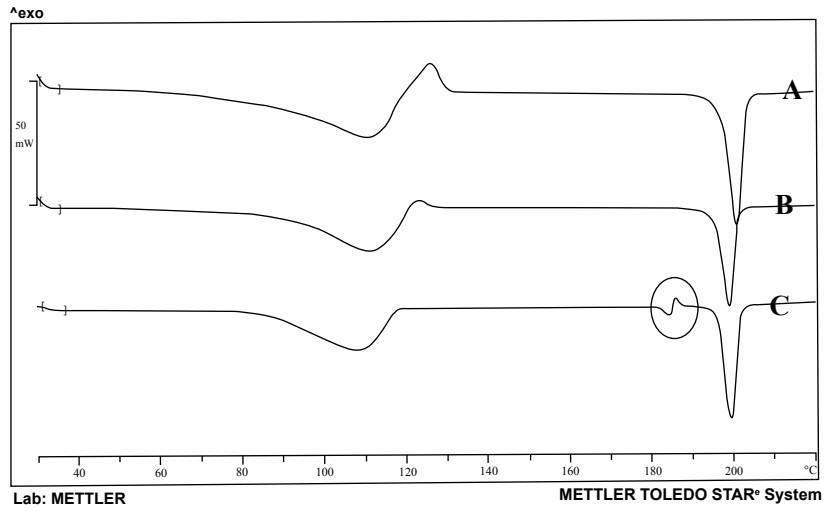

Figure 4: Olanzapine hydrate form. Thermogram profile of the hydrate obtained: A) from ethyl acetate saturated with water; B) from toluene saturated with water C) from acetone containing $10 \% \mathrm{v} / \mathrm{v}$ water. 
toluene mixtures and dichloromethane. The methods were proposed by Reutzel-Edens et al. [4] and were repeated here for comparison: the forms obtained were thus identified as Di-hydrates B, E; and higher hydrate respectively.

The sample obtained by precipitation from ethyl acetate /toluene $(1: 8.3 \mathrm{v} / \mathrm{v})$ by addition of water, after air-drying at room temperature (Di-hydrate B, according to [4]), shows a thermogram (Figure 6A) with the de-hydration endotherm at higher temperature with respect to that shown in Figure 3A and the melting endotherm of the form I at $197^{\circ} \mathrm{C}$ was present. Moreover the endotherm appears splitted (Figure 6A) and this fact is confirmed by the thermogravimetric profile (Figure $6 \mathrm{~B}$ ) showing at least three flexes that suggest a stepwise release of the crystallization molecules: range $14.13 \%\left(35-97^{\circ} \mathrm{C}\right.$; range $22.15 \%\left(97-121^{\circ} \mathrm{C}\right)$; range $32.18 \%\left(121-144^{\circ} \mathrm{C}\right)$. Changing the mixed solvent ratio, the thermogram of the precipitate (Di-hydrate E according to [4]) shows also endotherms partially fused, revealing also a thermogravimetric profile not continue displaying a flex at about $110^{\circ} \mathrm{C}$, related to the release of differently tied water molecules of crystallization (thermogram not shown).

In the conditions described by [4] to obtain the so-called higher hydrate, a dichloromethane solution of olanzapine was added with water. A large endotherm is evident in the thermogram of irregular profile that appears formed by two endotherms fused together. An exotherm is present peaking at about $120^{\circ} \mathrm{C}$; moreover a sharp signal of polymorph transition is present in the temperature range $170-190^{\circ} \mathrm{C}$ (Figure 7A).

The thermogravimetric profile (Figure 7B) indicates a flex at about $100^{\circ} \mathrm{C}$ and a total weight loss of $15.13 \%$, much higher than that expected from a simple Di-hydrate $(10.20 \%)$ that allows The term Higher hydrate for the samples obtained this way. Higher content (12.50\%) of crystallization water was also encountered in samples obtained by addition of water to olanzapine solution in acetone, in the range $5-10 \% \mathrm{v} / \mathrm{v}$.

While it was easily to measure these higher water percentages with the thermogravimetric analysis, it remains unresolved why it is so easy to load varying amount of water with different solvent/non-solvent processes and how could be accommodated the water molecules exceeding those of the common Di-hydrate form of olanzapine.

Structure of olanzapine: A crystal of olanzapine form I contains a

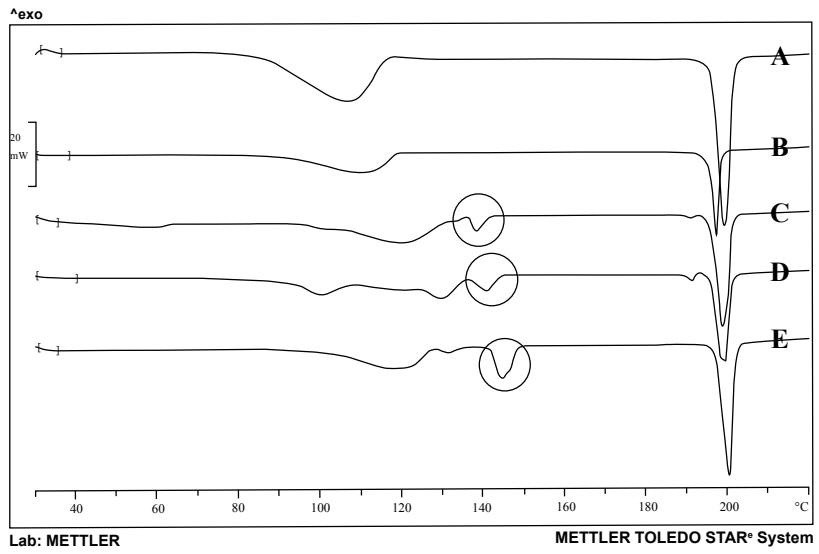

Figure 5: Thermogram profiles obtained from: A) acetone containing few drops of water; B) water containing $40 \% \mathrm{v} / \mathrm{v}$ methanol; C) water containing $60 \% \mathrm{v} / \mathrm{v}$ methanol; D) water containing $80 \% \mathrm{v} / \mathrm{v}$ methanol; E) pure methanol.

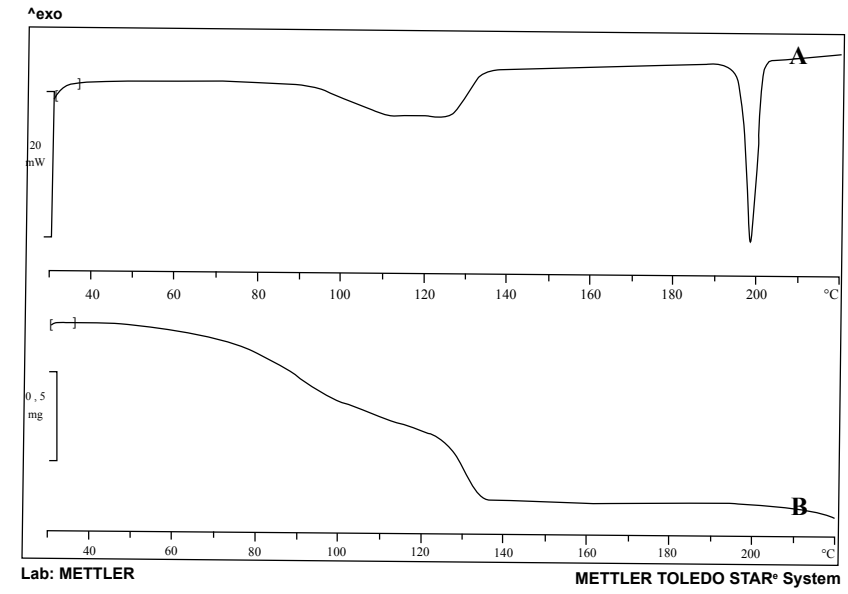

Figure 6: Thermogram $(A)$ and thermogravimetric $(B)$ profiles of the sample "Di-hydrate B" [4].

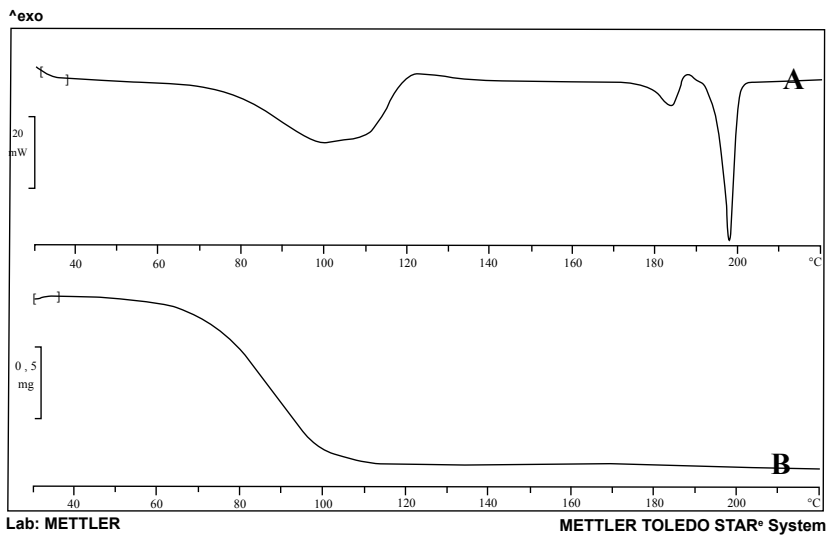

Figure 7: Thermogram $(A)$ and thermogravimetric $(B)$ profiles of the sample "Higher hydrate" [4].

structural block formed by piling of dimeric units staked into columns and connected and stabilized each other through internal NH.... N hydrogen bonds between the diazepine and piperazine rings or weak non-bonding interactions [2].

It is important to outline a few aspects of this organization of the solid state of olanzapine in order to interpret the experimental results.

The structure appears quite "rigid" in spite of the weak interactions involved in its stability, recognized by the relatively high melting point, associated to a relatively low melting $\Delta \mathrm{H}$. The rigidity of the crystal structure could explain also occurrence of two polymorph forms not easily in equilibrium each other; the two forms I and II are reported to be monotropically related and, though the transformation is associated only with a conformational change [4], it occurs only at high temperature, very close to the melting event, when the forces keeping the crystal lattice intact are almost completely released.

The structure results to be also very versatile, since the hydrogen bonds, responsible of the organization of the crystal can be mediated also by solvent molecules in the solvate, without any major alteration of it $[2-4,10]$. 
This structure leaves empty volumes that can be occupied by extra molecules in solvates, causing only distortion of the crystal lattice and subsequent increasing the intermolecular distances. However these volumes are not entered by water molecules from a humid atmosphere, in the absence of an external action (heat or solvent) that relaxes the crystal structure of olanzapine facilitating the entry and trapping of foreign molecules.

During heating the (hydrate/solvate) structure is forced to open to releasing the extra molecules contained at relatively low temperature (in the range $100-120^{\circ} \mathrm{C}$ ) and the original organization of the hydrate is temporarily lost: this event is described by the large endotherm found in every case; then, soon after the loss of the solvent molecules, the recovery of the original compact structure is documented by the exotherm. However after these steps it is possible the original organization could have been (at least partially) lost, resulting this fact in the appearance of unexpected peaks in the thermogram or of trace of form II, not previously present in the thermogram of the starting structure.

From this point of view thermal analysis, which often demonstrates to be quite invasive, just for this fact, highlights unexpected behaviour in the solid state of olanzapine hydrates and solvates and suggesting that the formation of the compacted structure, either during crystallization from solvents or after loss of solvent molecules following desolvation, is critical for the final organization. The easy insertion of solvent molecules inside the crystal lattice of olanzapine may result in a unforeseen multiplication of the structures that can be obtained for olanzapine, without being real polymorph forms or stoichiometric solvates or hydrates (especially when these are obtained by the mixed solvent method), but an often undefined mixtures of few canonical forms (form I and II; unsolvated and/or solvated form of the solvent used for crystallization). These ideas agree with the statement that "the many different structures in which olanzapine has been reported to crystallizes probably differ only slightly in their packing arrangement, irrespective of the solvate content" [3]. These forms could be the result of rapid precipitation or during desolvation on heating when the compact organization of olanzapine in the solid state is altered by the rapidity of the processes.

\section{Conclusion}

The final suggestion to obtain a pure form, when crystallizing olanzapine from any solvent, is: a) to check the possible formation of solvate by DSC; b) to heat the sample in the oven at $40-50^{\circ} \mathrm{C}$ until a simple thermogram is obtained (without any endotherm at low temperature); c) to evaluate the form (I or II) left by the desolvation process; d) to estimate, through the melting $\Delta \mathrm{H}$ value, the crystallinity of the final form.

\section{References}

1. Wawrzycka-Gorczyca I, Borowski P, Osypiuk-Tomasik J, Mazur L, Koziol AE (2007) Crystal structure of olanzapine and its solvates. Part 3. Two and threecomponent solvates with water, ethanol, butan-2-ol and dichloromethane. J Mol Struct 830: 188-197.

2. Wawrzycka-Gorczyca I, Koziol AE, Gliceb M, Cybulskib J (2004) Polymorphic form II of 2-methyl-4-(4-methyl-1-piperazinyl)-10H-thieno-[2,3-b][1,5]benzodiazepine. Acta Cryst E60: 66-68.

3. Ayala AP, Siesler HW, Boese R, Hoffmann GG, Polla GI, et al. (2006) Solid state characterization of olanzapine polymorphs using vibrational spectroscopy. Int J Pharm 326: 69-79.

4. Reutzel-Edens SM, Bush JK, Magee PA Stephenson GS, Byrn SR (2003) Anhydrates and Hydrates of Olanzapine: Crystallization, solid-state characterization, and structural relationships. Crystal Growth \& Design 3 897-907.

5. Bunnell CA, Hotten TM, Larsen SD, Tupper DE (1997) Process and solvate of 2 methyl-thieno-benzodiazepine. US Patent 5,703,232.

6. Blanco M, Coello J, Iturriaga H, Maspoch S, Perez-Maseda C (2000) Determination of polymorphic purity by near infrared spectrometry. Anal Chim Acta 407: 247-254.

7. Tiwari M, Chawla G, Bansal AK (2007) Quantification of olanzapine polymorph using powder X-ray diffraction technique. J Pharm Biomed Anal 43: 865-872.

8. Kolodziejski W, Herold J, Kuras M, Wawrzycka-Gorczyca I, Koziol AE (2011) Kinetics of $1 \mathrm{H}-13 \mathrm{C}$ NMR cross-polarization in polymorphs and solvates of the antipsychotic drug olanzapine. Solid State Nucl Magn Reson 39: 41-46.

9. Polla GI, Vega DR, Lanza H, Tombari DG, Baggio R, et al. (2005) Therma behaviour and stability in Olanzapine. Int J Pharm 301: 33-40.

10. Bunnel CA (1998) Olanzapine polymorph crystal form. US Patent

11. Hendriksen BA, Felix MV, Bolger MB (2003) The composite solubility versus $\mathrm{pH}$ profile and its role in intestinal absorption prediction. AAPS PharmSci 5: E4.

12. Wawrzycka-Gorczyca I, Mazur L, Koziol AE (2004) 2-Methyl-4-(4-methyl-1 piperazinyl)-10H-thieno[2,3-b] [1,5]benzodiazepine methanol solvate. Acta Crystallogr Sect E Struct 60: O69-071.

13. Capuano B, Crosby I, Fallon GD, Lloyd EJ, Yuriev E, et al. (2003) 2-Methyl4-(4-methylpiperazin-1-yl)-10H-thieno[2,3-b] [1,5]benzodiazepine methano solvate monohydrate. Acta Crystallogr Sect E 59: 01367-01369. 